\title{
Arthroscopically assisted anterior cruciate ligament reconstruction with bone-patellar tendon-bone autograft without wound drainage: short- to middle-term outcome
}

\author{
Dariusz Witoński ${ }^{1}$, Rafał Kęska ${ }^{2}$, Rafał Cyranowski ${ }^{3}$, Przemysław T. Paradowski ${ }^{4-6}$ \\ ${ }^{1}$ Social Academy of Science, Lodz, Poland \\ ${ }^{2}$ Department of Orthopaedics, University Clinical Hospital WAM, Lodz, Poland \\ ${ }^{3}$ Department of Orthopaedics and Traumatology, St. Raphael Hospital, Czerwona Gora, Poland \\ ${ }^{4}$ Department of Orthopaedics, Sunderby Central Hospital of Norrbotten, Luleå, Sweden \\ ${ }^{5}$ Department of Orthopaedics and Traumatology, University Clinical Hospital WAM, Lodz, Poland \\ ${ }^{6}$ Clinical Epidemiology Unit, Orthopaedics, Department of Clinical Sciences, Lund University, Lund, Sweden
}

Videosurgery Miniinv 2016; 11 (2): 76-82 DOI: $10.5114 /$ wiitm.2016.60044

\begin{abstract}
Introduction: Several studies have suggested that anterior cruciate ligament reconstruction (ACLR) without wound drainage has no impact on long-term follow-up.

Aim: To investigate a prospective patient series as measured by the patient-administered disease-specific questionnaire Knee injury and Osteoarthritis Outcome Score (KOOS).

Material and methods: The study included 101 consecutive patients (71 men and 30 women) with a mean age of 30 years (SD 10, range: 15-62 years), who had undergone primary single incision arthroscopic bone-patellar tendon-bone autograft (BPTB) ACLR without wound drainage. All patients completed KOOS questionnaires, preoperatively and at a mean follow-up of 1.4 years (range: 0.4-3.4). Satisfactory clinical outcome (function recovery - FR) was defined as the lower threshold for the $95 \% \mathrm{Cl}$ of 18-34-year old males and corresponded to a KOOS score > 90 for Pain, 84 for Symptoms, 91 for Activities of Daily Living (ADL), 80 for Sports/Recreation, and 81 for Quality of Life (QOL). A non-satisfactory result was defined as treatment failure (TF) and corresponded to a QOL score $<44$.

Results: All patients achieved $90^{\circ}$ of knee flexion on the first postoperative day and full extension 2 weeks postoperatively. A full range of motion was achieved in less than 6 weeks postoperatively. No postoperative complications were reported. Score improvement at follow-up was observed in the KOOS subscales Pain, Symptoms and ADL.Criteria for FR were fulfilled by 52\% of patients for Pain, $47 \%$ for Symptoms, $62 \%$ for ADL, 34\% for Sports/Recreation and $15 \%$ for QOL, whereas criteria for TF were fulfilled by $29 \%$ of patients.

Conclusions: The study demonstrated that the primary ACLRs without wound drainage did not have any negative impact for patient-reported recovery.
\end{abstract}

Key words: drainage, knee, anterior cruciate ligament, Knee injury and Osteoarthritis Outcome Score, arthroscopic anterior cruciate ligament reconstruction.

\section{Introduction}

Anterior cruciate ligament reconstruction (ACLR) is one of the most frequent orthopedic procedures. The operation was formerly done on an inpatient basis, but as medical technology progressed and surgical techniques improved, ACLRs have increasingly been performed as outpatient procedures [1, 2]. Daycare

\section{Address for correspondence}

Przemysław T. Paradowski MD, PhD, Department of Orthopaedics and Traumatology, University Clinical Hospital WAM,

113 Żeromskiego St, 90-549 Lodz, Poland, phone: +48 505724 726, e-mail: przemyslaw.t.paradowski@gmail.com 
ACLR has been reported to be safe, effective for pain control and not related to increased incidence of complications or readmissions to hospital [2]. However, in several orthopedic departments, patients undergoing ACLR stay at hospital for at least 2-3 days, due to wound drainage [1]. Several studies have shown no apparent advantage of drain use in ACLR [3, 4]. Nevertheless, there are surgeons who advocate the use of a wound drain in order to minimize the risk of limb swelling, deep vein thrombosis, intra-articular adhesions and joint stiffness [3, 5], while others, including the authors of the present study, feel that the use of a drain might increase the risk of infection or cause damage to the ACL graft and articular surfaces of the knee joint [4]. There is, however, a paucity of studies reporting that ACLR without a drain is superior to that with wound drainage [4] and, to our knowledge, there are no studies assessing the outcome of ACLR without a drain from the patient's perspective. The development of a number of validated, reliable and responsive self-assessment scores and the patient-relevant outcomes (PROs) for knee ligament injuries made it possible to monitor the outcome of surgical intervention [6]. The largest databases in which ACLR patients report outcome scores on the functioning of their knee using the commonly used PRO Knee injury and Osteoarthritis Outcome Score (KOOS) [7-9] are the National Knee Ligament Registries in Sweden, Norway and Denmark [10] and the MOON Register in the USA [11]. The Swedish ACL register shows that the percentage of subjects who are operated on on an outpatient basis appears to be more than $85 \%$ of the total number of operations and is slowly increasing [12]. One can suppose that subjects undergoing day surgery are operated on without the use of wound drainage. However, no separate results for those operated on with and without a drain are available.

\section{Aim}

Thus, the purpose of the study was to evaluate the clinical outcome of primary single incision arthroscopic bone-patellar tendon-bone autograft ACLR in a prospective patient series as measured by the patient-administered questionnaire KOOS.

\section{Material and methods}

\section{Study sample}

All patients who had undergone ACLR between January 2007 and November 2011 were identified by searching the surgical records at our department. In that period, 325 ACLR procedures were performed. All subjects underwent primary single incision arthroscopic bone-patellar tendon-bone autograft reconstruction. Exclusion criteria were: concomitant meniscal tears requiring suturing, chondral lesion that requires extensive chondroplasty, multiple ligamentous injuries. Inclusion and exclusion criteria were used to identify 101 patients who completed self-administered questionnaires evaluating their knee-specific symptoms and function preoperatively and during follow-up.

\section{Operative technique and rehabilitation}

The procedures were performed under subarachnoid anesthesia, in a bloodless field using a pneumatic tourniquet inflated to $250-270 \mathrm{~mm} \mathrm{Hg}$. All patients received deep vein thrombosis prophylaxis with subcutaneous dalteparin (Fragmin, Pfizer Inc., Woodcliff Lake, NJ, USA) 5000 IU/0.2 ml daily. Perioperative antibiotic prophylaxis was provided using a single dose of an antibiotic administered intravenously half an hour before the operation. The patient was placed in a supine position. The thigh was placed in a leg holder with knee unrestricted motion from full extension to $120^{\circ}$ of flexion. The central third bone-patellar tendon-bone graft was harvest through a midline skin incision between the patella and tibial tubercle. An oscillating saw was used to create 9 or $10 \mathrm{~mm} \times 20 \mathrm{~mm}$ bone plugs with a $9 \mathrm{~mm}$ to $10 \mathrm{~mm}$ wide tendon. The arthroscope was inserted using an antero-lateral portal. The working portal was medial to the patellar tendon. The knee was examined in a typical manner. If indicated, at that time, additional arthroscopic procedures such as meniscectomies, synovectomies, or shavings were performed. Remnants of the ruptured ACL were removed using a motorized full radius resector. Using a footprint of the native ACL, a femoral tunnel was first drilled through the antero-medial portal. Then the tibial tunnel was placed at an angle determined by the " $\mathrm{N}+7$ " formula to prevent graft extrusion or excessive recession [13]. After passing the graft, the graft bone plugs were stabilized in tunnels using titanium interference screws. The patellar as well as distal bony harvest sites were filled with an absorbable hemostatic gelatin sponge (Spongostan, Ferrosan Medical Devices A/S, Søborg, Denmark). The infrapatellar fat pad, soft tissues around the tibial tunnel and the subcutaneous tissues in the region 
of the skin incision were infiltrated with $20 \mathrm{ml}$ of $0.25 \%$ bupivacaine/epinephrine solution (Hospira Inc., Lake Forest, IL, USA). No drainage was used. The peritendinous tissue was closed but the tendon defect was left open. The skin incision was closed in layers. The tourniquet was released after applying a sterile dressing and soft-padded bandage. Cold therapy was provided immediately after the operation. On the first day after surgery, the patients were verticalized. Active full extension and flexion of the operated knee to the angle of $90^{\circ}$ were introduced. Patients were able to ambulate with mobility aids one day after the surgery with weight bearing of the operated limb "as tolerated". After the second week, unrestricted flexion was initiated/authorized. Crutches could be used for comfort as long as the patient desired, but most of them did not use them for more than 3 weeks. A hinged knee brace was not used. After a full range of motion was achieved/restored, strengthening exercises were added. Usually patients were allowed to return to sports activities after the sixth month, once they had regained their agility, strength and coordination.

\section{Clinical assessment}

All subjects underwent a clinical evaluation, including the range of motion in the operated knee, wound healing complications, infections, the number of aspirations for hemarthrosis and occurrence of limb swelling.

\section{Disease-specific questionnaire}

The Knee injury and Osteoarthritis Outcome Score was used. KOOS is a 42-item self-administered knee-specific questionnaire that was developed for short- and long-term follow-up studies of knee injuries and knee osteoarthritis (OA), and is commonly used to evaluate the effect of orthopedic surgery, including ACLR $[7,8,14]$. The KOOS scale contains five subscales: Pain, other Symptoms, Activities of Daily Living (ADL), Sports and Recreation and Quality of Life (QOL). A separate score ranging from 0 to 100 , where 100 represents the best result, is calculated for each subscale [9]. The KOOS has already been culturally adapted in Polish and validated for ACLR patients [15].

Participants were asked to complete KOOS questionnaires twice, preoperatively and at a routine follow-up.

\section{Outcome measures}

The primary outcome was defined as a change from baseline to follow-up assessment in the average score for all KOOS subscales, covering Pain, Symptoms, ADL Function, Sports and Recreation Function, and QOL, with scores ranging from 0 to 100 (worst to best) [9]. Secondary outcomes included results on all five KOOS subscales and an analysis of functional recovery and treatment failure.

\section{Functional recovery and treatment failure}

Functional recovery (FR) level was defined as the lower threshold for the $95 \% \mathrm{Cl}$ of 18-34-year-old males from the Swedish reference population [16], which represents a KOOS score above: 90 for the subscale Pain, 84 for Symptoms, 91 for ADL Function, 80 for Sports and Recreation Function, and 81 for QOL, respectively. Treatment failure (TF) was defined as the KOOS subscale QOL score < 44 [17].

\section{Clinically significant difference}

The minimal perceptible clinical improvement $(\mathrm{MPCl})$ represents the difference on the measurement scale associated with the smallest change in the health status that could be detected by the patient. A level of 10 points or more on a $0-100$ scale was established as a cut-off representing a clinically significant difference $[18,19]$.

\section{Ethics}

The study was approved by the ethics committee (approval no. RNN/190/07/KB). Informed written consent was obtained from all subjects who participated in the study.

\section{Statistical analysis}

Continuous outcomes are given as mean [standard deviation, SD] values. We used the Wilcoxon signed ranks test for assessment of comparisons between the groups. Binary data in $2 \times 2$ tables were evaluated by Fisher's exact test. We calculated the standardized response mean (SRM) by dividing average score change of KOOS subscales by SD of score change and effect size (ES) by dividing score change by baseline SD. Values of $p<0.05$ were considered significant. All analyses were performed with the SPSS for Windows 15.0 software package (SPSS Inc., Chicago, IL, USA). 


\section{Results}

\section{Study sample}

The study sample consisted of one hundred and one consecutive patients (71 men and 30 women) with a mean age of 30 years (median: 27, range: 15-62 years). No significant differences between the age of men and women was observed (mean: 28 (8) vs. 32 (11) years, $p=0.06$ ). The mean follow-up time was 1.4 years (range: $0.4-3.4$ ). The patient characteristics are given in Table I.

\section{Clinical assessment}

All patients achieved $90^{\circ}$ of knee flexion on the first postoperative day and full extension 2 weeks postoperatively. A full range of motion was achieved/restored in less than 6 weeks postoperatively. No postoperative complications were reported in the study group.

\section{Patient-relevant outcome}

Patients reported statistically significant improvements in scores from before surgery to follow-up in the KOOS subscales Pain, Symptoms and ADL Function. We did not observe any significant im-
Table I. Subject characteristics

\begin{tabular}{|lc|}
\hline \multicolumn{2}{|l|}{ Characteristics } \\
\hline$N$ (\% women) & $101(30)$ \\
\hline Age, mean (SD) [years]: & $29.6(9.4)$ \\
\hline ACL surgery & $31.0(9.4)$ \\
\hline Follow-up assessment & $1.4(0.6)$ \\
\hline Time to follow-up & \\
\hline
\end{tabular}

provement in the KOOS subscales Sports and Recreation Function and QOL (Table II).

We found substantial changes when the individual patients' scores before surgery and at follow-up were compared. The number of those who improved following ACLR was greater than the number of those who deteriorated in every KOOS subscale. Most patients improved in the subscales Sports and Recreation and QOL. The number of those who deteriorated was largest in the subscales Symptoms and Sports and Recreation (Table III).

\section{Functional recovery and treatment failure}

Twelve (12\%) patients out of 101 who had undergone ACLR fulfilled the criteria of FR in all KOOS subscales. The number of subjects who scored over

Table II. KOOS subscale scores before surgery and at follow-up

\begin{tabular}{|c|c|c|c|c|c|}
\hline \multirow[t]{2}{*}{ KOOS subscales } & \multicolumn{2}{|c|}{ Mean KOOS score $( \pm S D)$} & \multirow[t]{2}{*}{$P$-value } & \multirow{2}{*}{$\begin{array}{l}\text { Standardized } \\
\text { effect size }\end{array}$} & \multirow{2}{*}{$\begin{array}{l}\text { Standardized } \\
\text { response mean }\end{array}$} \\
\hline & Before surgery & At follow-up & & & \\
\hline Pain & $83.1(14.9)$ & $85.5(14.5)$ & 0.006 & 0.16 & 0.14 \\
\hline Symptoms & $76.5(17.4)$ & $79.5(16.5)$ & 0.001 & 0.17 & 0.15 \\
\hline$A D L$ & $87.0(14.3)$ & $90.2(13.1)$ & 0.007 & 0.22 & 0.20 \\
\hline Sports/Rec & $52.0(27.9)$ & $64.0(27.9)$ & 0.07 & 0.43 & 0.34 \\
\hline QOL & $45.8(22.1)$ & $57.4(23.7)$ & 0.18 & 0.52 & 0.38 \\
\hline
\end{tabular}

Table III. Absolute number of subjects (\%) who reported KOOS score change at follow-up compared with before surgery. Cut-off for minimal clinical change was set at 10 points

\begin{tabular}{|c|c|c|c|}
\hline \multirow[t]{2}{*}{ KOOS subscales } & \multicolumn{3}{|c|}{$\begin{array}{c}\text { Patients }(N=101) \\
n(\%)\end{array}$} \\
\hline & Improvement & No change & Deterioration \\
\hline Pain & $27(27)$ & $56(55)$ & 18 (18) \\
\hline Symptoms & $33(33)$ & $42(42)$ & $26(26)$ \\
\hline ADL & $23(23)$ & $65(64)$ & $13(13)$ \\
\hline Sports/Rec & $59(58)$ & $15(15)$ & $27(27)$ \\
\hline QOL & $53(52)$ & $25(25)$ & $23(23)$ \\
\hline
\end{tabular}


the threshold for functional recovery in separate KOOS subscales was: 52 (52\%) for Pain, $47(47 \%)$ for Symptoms, 62 (61\%) for ADL, 34 (34\%) for Sports and Recreation and 15 (15\%) for QOL.

We identified 29 (29\%) patients who obtained a score of $<44$ in the KOOS subscale QOL, thus fulfilling the criteria of TF. Out of those who had TF, four subjects improved at follow-up by $\geq 10$ points, two subjects deteriorated by $\geq 10$ points and 23 subjects did not change.

\section{Discussion}

The analysis of the primary outcome measure for patient follow-up in the current study demonstrates an improvement in the KOOS subscales Pain, Symptoms and ADL Function and no significant improvement in two KOOS subscales: Sports and Recreation Function and Quality of Life.

Although the follow-up outcomes are comparable to those reported by others, one would certainly anticipate a little larger and statistically more significant differences between the preoperative stage and follow-up outcome. These findings are, however, not unexpected. Our results in the subscales Sports and Recreation Function and Quality of Life were inferior to those of others [12, 20, 21], partly due to the relatively small study group and partly due to the criteria of patient selection [22]. As our qualification policy is quite liberal, we operated on even such patients who scored relatively well in the KOOS subscales before the ACLR. These patients usually did not change clinically or even deteriorated at follow-up assessment. By contrast, those subjects who improved to the greatest extent usually had the lowest scores following the preoperative rehabilitation. Thus, subjects with high preoperative scores might have made the ACLR results remarkably underestimated.

Our secondary outcome measure was to analyze the functional recovery and treatment failure. We found that about half of the patients undergoing ACLR achieved functional recovery in the KOOS subscales Pain and Symptoms. The percentage of those who recovered in the subscale ADL Function was even higher (62\%). The same ADL Function subscale included the smallest number of patients who deteriorated. It has previously been shown, however, that for younger and active patients, the KOOS subscale ADL Function is less sensitive than other subscales and can be omitted $[23,24]$. Thus, an as- sessment of the clinical outcome aside from the ADL Function score was suggested [23]. The percentage of patients who scored $<44$ in the KOOS subscale of knee-related $\mathrm{QOL}$ and thus fulfilled the criteria for treatment failure was relatively high, but similar to that reported in other studies [12]. Since it has been reported that at 1-year follow-up the ACL is not fully functional, one can expect that the QOL score would be higher the next year and beyond.

In addition, our study revealed that ACLR can be carried out without wound drains. This finding seems to be important in view of the fact that this procedure is increasingly performed as day-case surgery $[5,12,25]$.

However, Müller-Rath et al. evaluated the current standards of perioperative management following outpatient arthroscopic surgery of the knee in Germany and revealed that suction drainage was applied by $36 \%$ of surgeons regularly and by $45 \%$ occasionally. A drain was left for one day by $79 \%$ of surgeons, while $11 \%$ used it only for several hours [26].

We found no tendency to any local complications such as perioperative bleeding, hematoma needing a puncture and aspiration, limb ischemia requiring special treatment or abnormal wound healing. El Khalifa et al. in a prospective randomized control trial (RCT) of ACLR with four-strand hamstring autograft showed that patients with a drain suffered less pain and hemarthrosis than those without a drain. The range of movements in the first week following the operation was better in the drain group than in subjects without a drain. The drain was removed on the first postoperative day [27]. In another RCT, Dhawan et al. found that the use of a drain after ACLR with a BPTB autograft provided no benefit in terms of the range of motion, effusion, or pain in the early postoperative period [28]. No functional differences in subjects undergoing ACLR with BPTB autograft at 6-month follow-up were reported by Straw et al. [5].

To our knowledge, the present study describes the largest series of patients to date who underwent ACLR without wound drainage. The study is also the first attempt to assess the clinical outcome of these patients with PROs. The strengths of our study also include prospective evaluation as well as standardization of hospitalization procedures with the participation of the same operating team and the use of an identical rehabilitation regimen, objective evaluation by an indirect method assessing the range 
of motion in the operated joint and a subjective assessment using the KOOS questionnaire.

Our study findings must be interpreted considering the study limitations. First, the number of subjects does not entitle us to express an opinion about the strength of the obtained results. It is, however, sufficient for a statistical evaluation. Second, since we do not perform ACLR with drains, we cannot compare the results of these two approaches. Finally, our patient follow-up endpoint was about one year after surgery. Since we know that at that time the ACL tends not to be fully functional [12], a longer follow-up period is needed to report more reliable results.

\section{Conclusions}

The present study demonstrated that primary single incision, arthroscopic bone-patellar tendon-bone autograft ACLR without wound drainage, did not have any negative impact for patient-reported recovery.

\section{Conflict of interest}

The authors declare no conflict of interest.

\section{References}

1. Krywulak SA, Mohtadi NG, Russell ML, Sasyniuk TM. Patient satisfaction with inpatient versus outpatient reconstruction of the anterior cruciate ligament: a randomized clinical trial. Can J Surg 2005; 48: 201-6.

2. Valkering KP, van Bergen CJ, Buijze GA, et al. Pain experience and functional outcome of inpatient versus outpatient anterior cruciate ligament reconstruction, an equivalence randomized controlled trial with 12 months follow-up. Knee 2015; 22: 111-6.

3. McCormack RG, Greenhow RJ, Fogagnolo F, Shrier I. Intra-articular drain versus no drain after arthroscopic anterior cruciate ligament reconstruction: a randomized, prospective clinical trial. Arthroscopy 2006; 22: 889-93.

4. Clifton R, Haleem S, McKee A, Parker MJ. Closed suction surgical wound drainage after anterior cruciate ligament reconstruction: a systematic review of randomised controlled trials. Knee 2007; 14: 348-51.

5. Straw R, Colclough K, Geutjens GG. Arthroscopically assisted ACL reconstruction. Is a drain necessary? Knee 2003; 10: 283-5.

6. Hill GN, O'Leary ST. Anterior cruciate ligament reconstruction: the short-term recovery using the Knee Injury and Osteoarthritis Outcome Score (KOOS). Knee Surg Sports Traumatol Arthrosc 2013; 21: 1889-94.

7. Miller MD, Hinkin DT. The $N+7$ rule for tibial tunnel placement in endoscopic anterior cruciate ligament reconstruction. Arthroscopy 1996; 12: 124-6.

8. Roos EM, Roos HP, Lohmander LS, et al. Knee injury and osteoarthritis outcome score (KOOS) - development of a self-admin- istered outcome measure. J Orthop Sports Phys Ther 1998; 28 : 88-96.

9. Roos EM, Roos HP, Ekdahl C, et al. Knee injury and Osteoarthritis Outcome Score (KOOS) - validation of a Swedish version. Scand I Med Sci Sports 1998; 8: 439-48.

10. Knee injury and Osteoarthritis Outcome Score. http://www. koos.nu/index.html

11. Granan LP, Forssblad M, Lind M, Engebretsen L. The Scandinavian ACL registries 2004-2007: baseline epidemiology. Acta Orthop 2009; 80: 563-7.

12. Wright RW, Dunn WR, Amendola A, et al. Risk of tearing the intact anterior cruciate ligament in the contralateral knee and rupturing the anterior cruciate ligament graft during the first 2 years after anterior cruciate ligament reconstruction. A prospective MOON cohort study. Am J Sports Med 2007; 35: 1131-4.

13. Swedish ACL Register. Annual Report 2014. http://www.artroclinic.se/info/rapport2014en.pdf

14. Roos EM, Lohmander LS. The Knee injury and Osteoarthritis Outcome Score (KOOS): from joint injury to osteoarthritis. Health Qual Life Outcomes 2003; 1: 64.

15. Paradowski PT, Witoński D, Kęska R, Roos EM. Cross-cultural translation and measurement properties of the Polish version of the Knee injury and Osteoarthritis Outcome Score (KOOS) following anterior cruciate ligament reconstruction. Health Qual Life Outcomes 2013; 11: 107.

16. Paradowski PT, Bergman S, Sunden-Lundius A, et al. Knee complaints vary with age and gender in the adult population. Population-based reference data for the Knee injury and Osteoarthritis Outcome Score (KOOS). BMC Musculoskelet Disord 2006; 7: 38.

17. Barenius B, Forssblad M, Engström B, Eriksson K. Functional recovery after anterior cruciate ligament reconstruction, a study of health-related quality of life based on the Swedish National Knee Ligament Register. Knee Surg Sports Traumatol Arthrosc 2013; 21: 914-27.

18. Ehrich EW, Davies GM, Watson DJ, et al. Minimal perceptible clinical improvement with the Western Ontario and McMaster Universities osteoarthritis index questionnaire and global assessments in patients with osteoarthritis. J Rheumatol 2000; 27: 2635-41.

19. Paradowski PT, Englund M, Roos EM, Lohmander LS. Similar group mean scores, but large individual variations, in patient-relevant outcomes over 2 years in meniscectomized subjects with and without radiographic knee osteoarthritis. Health Qual Life Outcomes 2004; 2: 38.

20. Lind M, Menhert F, Pedersen AB. The first results from the Danish $A C L$ reconstruction registry: epidemiologic and 2 year follow-up results from 5,818 knee ligament reconstructions. Knee Surg Sports Traumatol Arthrosc 2009; 17: 117-24.

21. Norwegian ACL Register. Annual Report 2013. http://www. kvalitetsregistre.no/resultater/muskel-skjelett-og-bindevev/ nasjonalt-korsbaandregister/resultater/

22. Szmigielska A, Paradowski PT. Assessment of selection criteria for anterior cruciate ligament reconstruction. Health Care Curr Rev 2013; 1: 107.

23. Frobell RB, Roos EM, Roos HP et al. A randomized trial of treatment for acute anterior cruciate ligament tears. N Engl J Med 2010; 363: 331-42. 
24. Paradowski PT, Kęska R, Witoński D. Does concomitant meniscectomy affect medium-term outcome of anterior cruciate ligament reconstruction? A preliminary report. Arch Med Sci 2014; 10: 992-8.

25. Carvalho Júnior LH, Denaro MF, Gonçalves TJ, et al. The use of suction drain after anterior cruciate ligament reconstruction. Rev Bras Ortop 2006; 41: 211-6.

26. Müller-Rath R, Ingenhoven E, Mumme T, et al. Perioperative management in outpatient arthroscopy of the knee joint. Z Orthop Unfall 2010; 148: 282-7.

27. El Khalifa T, Al Mahozi A, Dhaif B. Intra-articular drain versus no drain after arthroscopic anterior cruciate ligament reconstruction: a randomized prospective clinical trial. Bahrain Med Bull 2008, 30: 9-11.

28. Dhawan A, Doukas WC, Papazis JA, Scoville CR. Effect of drain use in the early postoperative period after arthroscopically as sisted anterior cruciate ligament reconstruction with bone-patellar tendon-bone graft. Am J Sports Med 2003; 31: 419-24.

Received: 16.09.2015, accepted: 11.04.2016. 